\title{
Copper vapor laser drilling of copper, iron, and titanium foils in atmospheric pressure air and argon
}

\author{
J. S. Lash and R. M. Gilgenbach \\ Intense Energy Beam Interaction Laboratory, Nuclear Engineering Department, The University of \\ Michigan, Ann Arbor, Michigan 48109-2104
}

(Received 19 March 1993; accepted for publication 27 July 1993)

\begin{abstract}
A copper vapor laser ( 511 and $578 \mathrm{~nm}$ ) is used to drill submillimeter diameter holes in $0.025-$ $0.127 \mathrm{~mm}$ thick foils of copper, iron, and titanium. Foils are machined in atmospheric pressure air and argon. The laser is repetitively pulsed at $10 \mathrm{kHz}$ with a per pulse energy of $0.5 \mathrm{~mJ}$ giving an average power of $5 \mathrm{~W}$ at the sample surface for a pulse width of $40 \mathrm{~ns}$. A $p-i-n$ photodiode and a photomultiplier tube detector are connected to a digital-display timing circuit that records the number of incident laser pulses used to drill through the sample. The number of pulses is converted to an average drilling time and can provide an estimate for the average laser energy used to drill the hole. Typical data for all three materials with a per-pulse fiuence of $0.7 \mathrm{~J} / \mathrm{cm}^{2}$ ranged from 0.1 to $500 \mathrm{~s}$ to produce holes of $\sim 0.3 \mathrm{~mm}$ diameter. Drilling times decreased in some cases by an order of magnitude when machining in air. This is attributed to the increased laser absorption of the metal-oxide layer formed in air and was especially noticeable with titanium. A continuous wave thermal model is used to compare experimental data as well as verify the thermal machining mechanism.
\end{abstract}

\section{INTRODUCTION}

Drilling of materials with pulsed and continuous-wave laser light has important applications in manufacturing. The well-known use of the $\mathrm{CO}_{2}$ laser $(10.6 \mu \mathrm{m})$ has been the subject of much study for machining applications. ${ }^{1}$ Nd:YAG lasers have been used in turbine blade drilling in the aerospace industry ${ }^{2}$ while a number of different types of lasers have been employed for microelectronics drilling applications. ${ }^{3-5}$ Recently, excimer lasers operating at UV wavelengths have been studied for their use in the ablation of a wide range of materials. ${ }^{4}$ These applications typically operate with high per-pulse energy $(1-40 \mathrm{~J})$ and low repetition rates $(5-10$ pulses/s). This article reports the use of a high average power, commercially available, repetitively pulsed copper vapor laser. The low per-pulse energy of 0.5 $\mathrm{mJ}$ combined with a high repetition rate of $10 \mathrm{kHz}$ gives an average power of $5 \mathrm{~W}$ at the sample surface for the 511 and $578 \mathrm{~nm}$ wavelengths with a 40 ns pulse width. It has been shown that high repetition rate pulsed lasers have increased laser penetration efficiency over that obtained with continuous-wave lasers of similar power. ${ }^{6}$ The green $(511$ $\mathrm{nm})$ and yellow ( $578 \mathrm{~nm}$ ) light of the copper vapor laser also have advantages over the infrared light of the $\mathrm{CO}_{2}$ laser due to the known high reflectivity ( $>95 \%$ ) of most metals to $10.6 \mu \mathrm{m}$ light. ${ }^{9}$ With iron and titanium, typical room-temperature reflectivities with green-yellow light are $\sim 40 \%-50 \% .^{7}$

With per-pulse fluences of $0.7 \mathrm{~J} / \mathrm{cm}^{2}, 0.3-\mathrm{mm}$-diam holes have been drilled in $0.025-0.127 \mathrm{~mm}$ thick copper, iron, and titanium foils in atmospheric air and argon environments. Typical drilling times ranged from 0.1 to $500 \mathrm{~s}$ with foils of $99.5 \%-99.995 \%$ purity. Considcrable effects were observed when machining in air compared to argon. Drilling times decreased in some cases by an order of magnitude when machining in air. This is attributed to the increased absorption of the metal-oxide layer formed in air and was especially noticeable with titanium. Drilling with the copper vapor laser is a thermal as opposed to a photoablative process. The thermal machining of the copper vapor laser is verified, along with confirming the validity of the experimental data by modeling the hole drilling process with a continuous-wave thermal model.

\section{EXPERIMENTAL CONFIGURATION}

A simplified experimental configuration is shown in Fig. 1. Sample foils are machined using an Oxford Laser Company copper vapor laser pulsed at $10 \mathrm{kHz}$ with a perpulse energy of $0.5 \mathrm{~mJ}$ giving an average power of $5 \mathrm{~W}$ at the sample surface for the 511 and $578 \mathrm{~nm}$ wavelengths with a 40 ns pulse width. Focusing the laser beam's rectangular profile with a $12 \mathrm{~cm}$ focal-length lens produced hole diameters of $0.3 \mathrm{~mm}$ drilled with a per-pulse fluence of $0.7 \mathrm{~J} / \mathrm{cm}^{2}$ or an average power density of $7 \mathrm{~kW} / \mathrm{cm}^{2}$. A combination vertical foil holder and gas chamber allowed machining in atmospheric pressure air versus argon environments. Samples studied consisted of $0.025-0.127 \mathrm{~mm}$ thick aluminum, copper, iron, and titanium foils of $99.5 \%-$ 99.995\% purity. Prior to drilling, all foils were lightly sanded with 600-grit sandpaper to achieve uniformity in the surface characteristics. This was necessary since the samples as obtained from the manufacturers had varying degrees of surface smoothness. Uniformity was desired since the coupling of the laser light to the material likely depends on the surface characteristics. The sanding also removed any existing surface oxides. The machining and timing measurement is initiated by opening a mechanically activated camera shuttcr. A fraction of the incident laser beam is deflected onto a $p-i-n$ photodiode detector by a quartz beam splitter.

Figure 2 shows an electrical schematic of the timing 


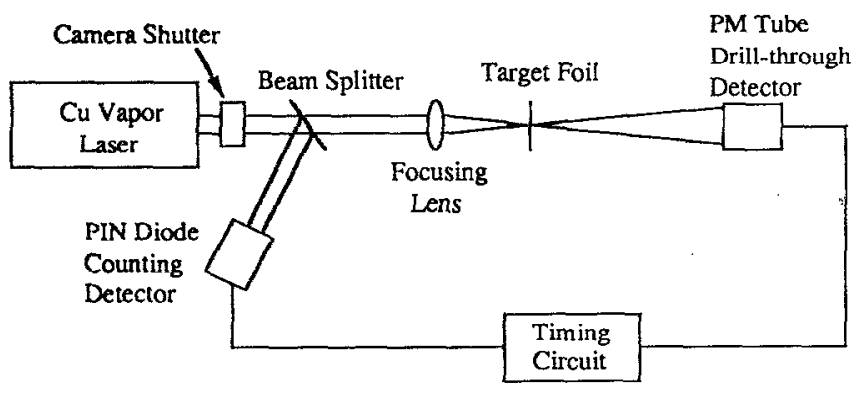

FIG. 1. Simplified drawing of the experimental configuration.

circuit. The $p-i-n$ photodiode pulse is input to an analogto-digital converter consisting of a precision monostable multivibrator (Motorola MC14538BCP) with an RC pulse length of one-half the laser pulse period. The digital pulse is then received by a digital counter (Harris ICM7217AIPI) driving a 4-digit numeric light-emitting diode (LED) display. An additional digital counter extends the display to 8 digits allowing drilling times between 0.0001 and $10000 \mathrm{~s}$ to be recorded with the $10 \mathrm{kHz}$ repetition rate. The first light through the foil indicating drill through is collected with a photomultiplier (PM) tube detector. Due to the high output impedance of the PM tube being comparable to the input impedance of the timer, a unity-gain inverting operational amplifier (Texas Instruments TLE2601CP) is used to reduce the loading of the photomultiplier tube. The PM tube signal triggers a resetable monostable multivibrator (Motorola MC14538BCP) with an RC pulse length greater than the laser pulse period, stopping the updating of the timing circuit display.

\section{EXPERIMENTAL RESULTS AND DISCUSSION}

Foils of titanium and iron were rapidly drilled through while drilling of copper required considerably longer times. Table I shows a summary of the average drilling times and average laser energies used to drill the holes for titanium and iron of two thicknesses in air and argon environments. Values shown are averages of 12-20 drilled holes while the errors are sample standard deviations. From Table $\Upsilon$ it is observed that with titanium the drilling time in air compared to argon was reduced by a factor of 4 with the 0.032 mm-thick titanium foil. Foils of 0.127 -mm-thick titanium were drilled through in air, but no machining was observed in argon. These differences are the result of the combined effects of optical property modification by formation of a metal-oxide layer at the foil surface and the exothermic nature of the oxidation reaction. Figure 3 shows optical microscope pictures of typical holes drilled in $0.032-\mathrm{mm}$ thick titanium foils in air and argon. The hole drilled in air shows substantial dark-blue discoloration beyond a region of grayish-white scale near the drill hole. The dark region is caused by formation of oxygen-deficient titaniumdioxide in the form of rutile while the grayish-white scale is rutile of true $\mathrm{TiO}_{2}$ stoichiometric composition. ${ }^{8}$ Extreme distortion of the irradiated area was also observed. The apparent darkened region around the hole in Fig. 3(b) is a result of this distortion. With an air background, the diffusion of oxygen into the surface causes embrittlement of the titanium. The rapid expansion and contraction during heating and cooling then results in the cracking and detachment of material around the drilled hole. With the aid of a microscope the stress cracking of the hole wall is observable. Figure 3(a) shows the hole dianeter to be substantially larger than the incident laser beam diameter. The rapid creation of titanium-dioxide combined with the low

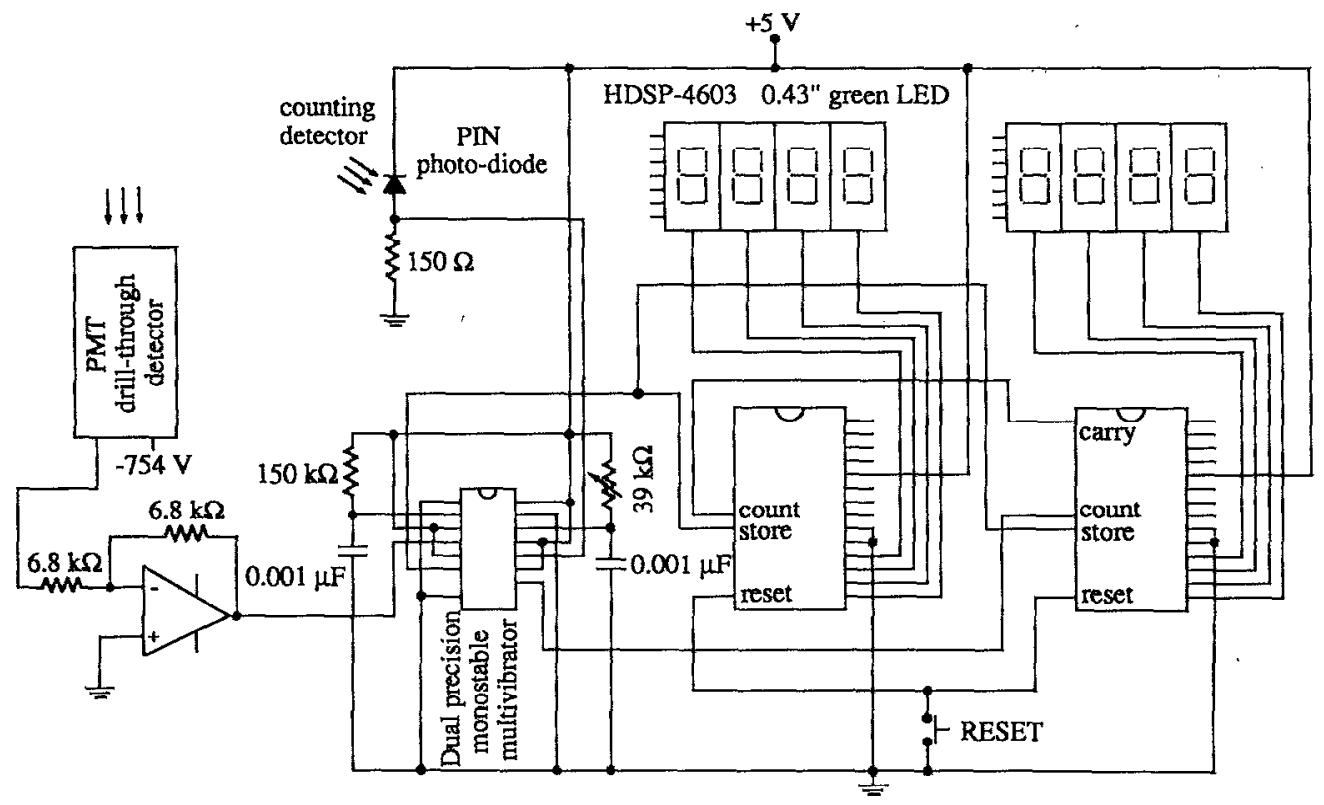

FIG. 2. Timing circuit electrical schematic. 
TABLE I. Average experimental drilling times and deposited energy for $\mathrm{Ti}$ and $\mathrm{Fe}$ foils.

\begin{tabular}{|c|c|c|c|c|c|}
\hline & & & & & argon \\
\hline & $\mathrm{mm})$ & Time (s) & Average laser energy ( $)$ & Time (s) & Average laset energy $(\mathrm{J})$ \\
\hline $\mathrm{Ti}$ & 0.032 & $0.63 \pm 7.6 \%$ & 3.2 & $2.42 \pm 44 \%$ & 12.1 \\
\hline & 0.127 & $140.20 \pm 67 \%$ & 701 & & achining \\
\hline $\mathrm{Fe}$ & 0.025 & $0.13 \pm 11 \%$ & 0.65 & $1.24 \pm 8.4 \%$ & 6.2 \\
\hline & 0.100 & $29.18 \pm 7.4 \%$ & 146 & & chining \\
\hline
\end{tabular}

thermal conductivity of the metal oxide resulted in little heat loss from the region around the irradiated area, allowing the temperature distribution above the vaporization point to extend beyond the irradiated area, enlarging the hole diameter.

The increase in absorptivity with temperature due to formation of titanium dioxide with incident $10.6 \mu \mathrm{m}\left(\mathrm{CO}_{2}\right.$ laser) light has been studied and is applied in oxygenassisted machining. ${ }^{9}$ With the copper vapor wavelengths ( 511 and $578 \mathrm{~nm}$ ), the absorptivity for titanium dioxide is not well known although the data in Table I suggest an increase in absorption over that of pure titanium. With large-scale machining where sizeable amounts of material are removed using jets of oxygen blowing on the target, the additional energy release from the exothermic oxidation reaction substantially aids in machining. ${ }^{9,11}$ In this experiment, with the small amount of material removed in the drill holes, calculations show that the extra oxidation reaction energy is small compared to the incident laser energy and has a minor effect on increasing the foil temperature.
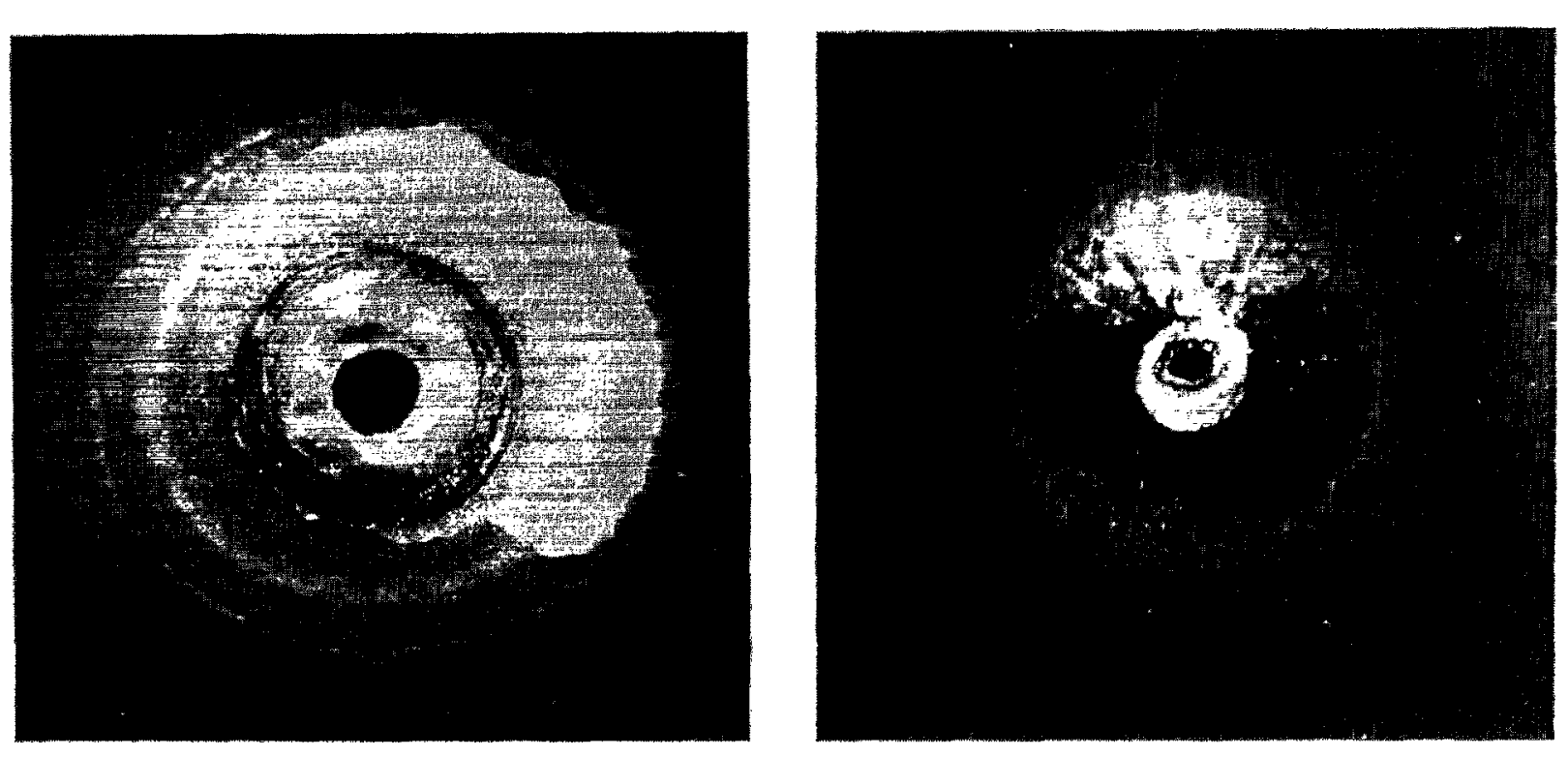

As in the case of titanium, drilling times for iron foils were substantially reduced when machining in air as opposed to argon. From Table I it is seen that for $0.025 \mathrm{~mm}$ iron, drilling in air reduced the drilling time from that in argon by an order of magnitude. Heating the iron in air causes oxidation of the surface resulting in the formation of a layer of iron oxide $\left(\mathrm{Fe}_{2} \mathrm{O}_{3}\right)$. This is observed as the darkened region around the drill hole in Fig. 4(a). The oxide layer increases the absorptivity of the foil resulting in decreased drilling times. In argon and in air, the irradiated area also shows distortion due to localized heating.

A simple model can be used to calculate drilling times for thermally thin foils. By thermally thin it is meant that there is no temperature gradient across the foil thickness where the laser is incident. The condition for thermally thin foils is met when the quantity $D^{2} / 4 k t$ is much less than 1 , where $D$ is the foil thickness, $k$ is the thermal diffusivity, and $t$ is the time scale considered. For foils of $\sim 0.025-0.127 \mathrm{~mm}$ thickness the above condition is easily satisfied, although for the thickest foils $(0.127 \mathrm{~mm})$ the model will produce less accurate results. The solution to

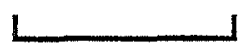

(a)

$1 \mathrm{~mm}$

(b)

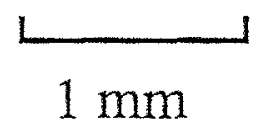

FIG. 3. Drilled holes in 0.032-mm-thick $T i$ in atmospheric pressure (a) air and (b) argon. 


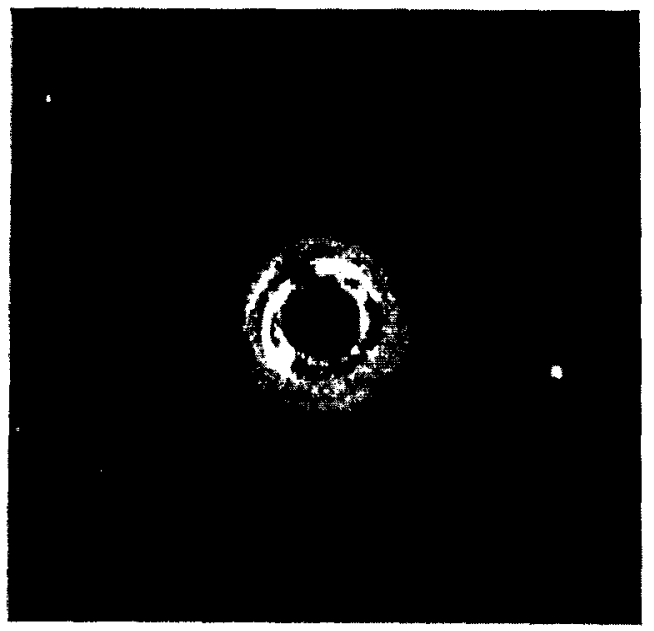

1

(a)
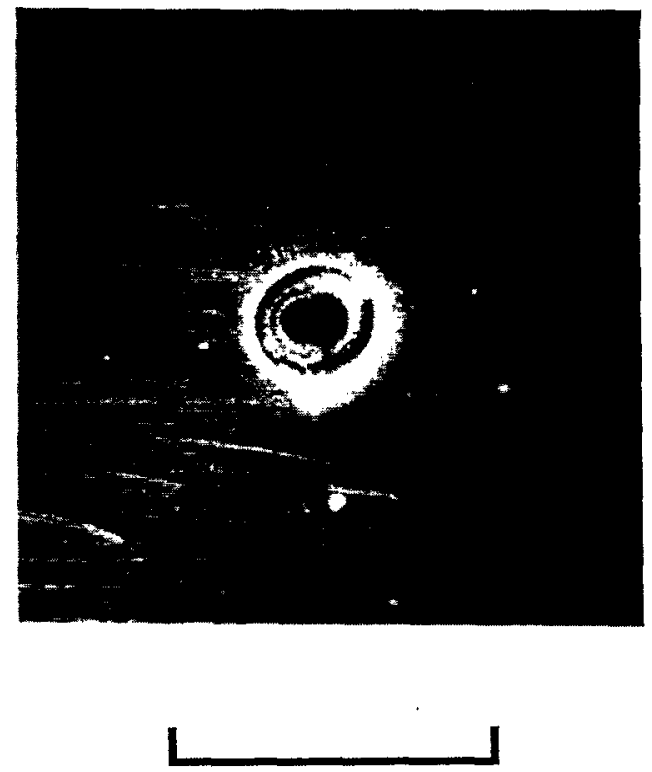

$1 \mathrm{~mm}$

FIG. 4. Drilled holes in 0.025 -mm-thick $\mathrm{Fe}$ in atmospheric pressure (a) air and (b) argon.

the heat flow equation for the above boundary conditions is well known. ${ }^{10}$ The temperature increase in degrees Kelvin as $a$ function of time at the center of the irradiation spot is given as

$$
\begin{aligned}
& T(t)=\frac{A P}{4 K D \pi} \int_{0}^{\tau} \frac{\exp \left(-\sigma t^{\prime}\right)}{t^{\prime}+1} d t^{\prime}, \\
& \sigma=\frac{H r^{2}}{4 K D}
\end{aligned}
$$

and

$$
\tau=\frac{8 \kappa t}{r^{2}},
$$

where $A$ is the temperature-averaged absorptivity ( $A=1$ $-R$, where $R$ is the reflectivity), $P$ the measured incident laser power, $\boldsymbol{K}$ the temperature-averaged thermal conductivity, $D$ the foil thickness, $\kappa$ the temperature-averaged thermal diffusivity, $r$ the beam radius at the foil, and $H$ the surface conductance of the material. ${ }^{11}$ An approximate value for $H$ with planar surfaces is $5 \times 10^{-4}$ $\mathrm{W} \mathrm{cm}^{-2} d \mathrm{~K}^{-1}$. The change in $H$ with temperature is small for the temperatures considered in this experiment and will be neglected. ${ }^{11}$

The model is employed to determine the time until the vaporization temperature is reached. Thermal conductivities and thermal diffusivities are thus averaged over the range from room temperature $(300 \mathrm{~K})$ to the vaporization temperature of the specific material. Except for $10.6 \mu \mathrm{m}$ light $\left(\mathrm{CO}_{2}\right.$ lasers) little work has been done on the temperature dependence of absorptivity for specific metals and specific optical wavelengths. Therefore only an estimate can be made for the average absorptivity over the temperature range considered. For $10.6 \mu \mathrm{m}$ light it is observed that for most metals, the absorptivity increases linearly with temperature and for the liquid phase is approximately a factor of 2 greater than the room-temperature $(300 \mathrm{~K})$ value. ${ }^{y}$ Since the vaporization temperatures of metals considered in this experiment are roughly a factor of 2 greater than the melting point temperatures, the average absorptivity over the temperature range considered is assumed to be approximately a factor of 1.75 greater than the roomtemperature absorptivity. Table II contains the roomtemperature $(300 \mathrm{~K})$ reflectivities ${ }^{7}$ for the copper vapor laser wavelengths ( 511 and $578 \mathrm{~nm}$ ) and the estimated temperature-averaged reflectivities for the sample materials used.

Calculations show that the additional heat for the phase transformation from solid to liquid is small compared to typical irradiation energies and will thus be neglected. Therefore the model provides an estimate for the hole-drilling time assuming the time to actually remove the material from the hole by vaporization is small. Since the drilling process is modified by the oxidation produced while machining in air and optical data for the oxides of the sample materials are unavailable, this thermal model

TABLE II. Target sample reflectivities (from Ref. 7) for copper vapor laser wavelengths and estimated temperature-averaged reflectivities.

\begin{tabular}{cccc}
\hline & \multicolumn{2}{c}{$\begin{array}{c}\text { Laser wavelength } \\
\text { reflectivities at } 300 \mathrm{~K}\end{array}$} & $\begin{array}{c}\text { Estimated temperature- } \\
\text { averaged reflectivities }\end{array}$ \\
\cline { 2 - 3 } & $511 \mathrm{~nm}$ & $\mathbf{5 7 8 \mathrm { nm }}$ & 0.57 \\
$\mathrm{Cu}$ & 0.60 & 0.75 & 0.23 \\
$\mathrm{Fe}$ & 0.57 & 0.56 & 0.12 \\
$\mathrm{Ti}$ & 0.48 & 0.51 & 0.90 \\
$\mathrm{Al}$ & 0.97 & 0.92 & \\
\hline
\end{tabular}




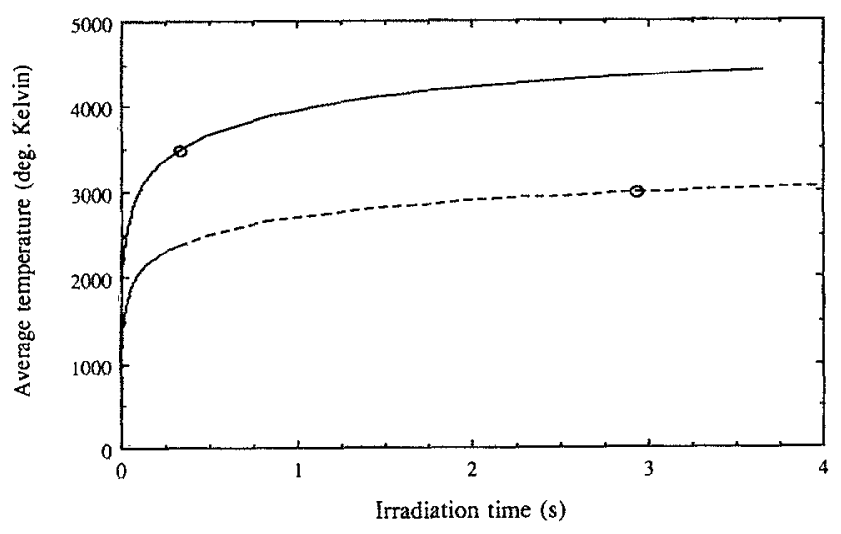

FIG. 5. Calculated average temperature as a function of irradiation time for iron 0.025 -mm-thick (dashed line) and titanium 0.032-mm-thick (solid line) foils in argon background gas. Estimated drill-through times are determined when vaporization temperature $(O)$ has been reached.

can only be compared to experimental values taken in the nonreactive argon background.

Figure 5 shows the calculated average temperature as a function of irradiation time from an initial temperature of $300 \mathrm{~K}$ for iron $(0.025 \mathrm{~mm})$ and titanium $(0.032 \mathrm{~mm})$. This is an average temperature since the laser is repetitively pulsed; however, the high repetition rate of $10 \mathrm{kHz}$ combined with the low per-pulse energy of $0.5 \mathrm{~mJ}$ will introduce a negligible modulation of the actual temperature as a function of irradiation time. Figure 5 shows that for titanium the calculated vaporization temperature is reached after $0.4 \mathrm{~s}$. The average experimental drill-through was determined to be $2.42 \mathrm{~s}$. For iron the model predicts $2.9 \mathrm{~s}$ to reach the vaporization temperature which is in reasonable agreement with the experimentally measured value of $1.24 \mathrm{~s}$. Further calculations for the $0.1-\mathrm{mm}$-thick iron and 0.127 -mm-thick titanium foils in argon showed that the melting-point temperature was never reached; this is confirmed by the observance of no machining on the foils as stated in Table I.

Differences between predicted and experimental values arise from surface effects as well as from assumptions made in deriving the model. One source of discrepancy is that the model only gives the time until the vaporization point is reached while the material must be removed from the irradiation area in order for the experimental drill through time to be recorded. Additionally, from the asymptotic form of the theoretical curve, small changes in the absorption or laser power change the temperature scaling, resulting in large time variations. The reasonable agreement between the experimental values and the values in Fig. 5 given the assumptions made, shows the validity of the model and confirms the thermal drilling mechanism. The thermal drilling process with a copper vapor laser has also been observed in the machining of polymers. ${ }^{12}$

Samples of copper foils showed drilling only in air. With an argon background no surface modification was observed at the irradiation spot; this is also confirmed by calculations using the thermal model. In air, average drilling times of 17.8 and $184.7 \mathrm{~s}$ were measured for thicknesses of 0.025 and $0.127 \mathrm{~mm}$, respectively with a $0.27 \mathrm{~mm}$ hole diameter. Figures $6(a)$ and $6(b)$ show typical holes drilled in copper of 0.025 and $0.127 \mathrm{~mm}$ thickness, respectively.
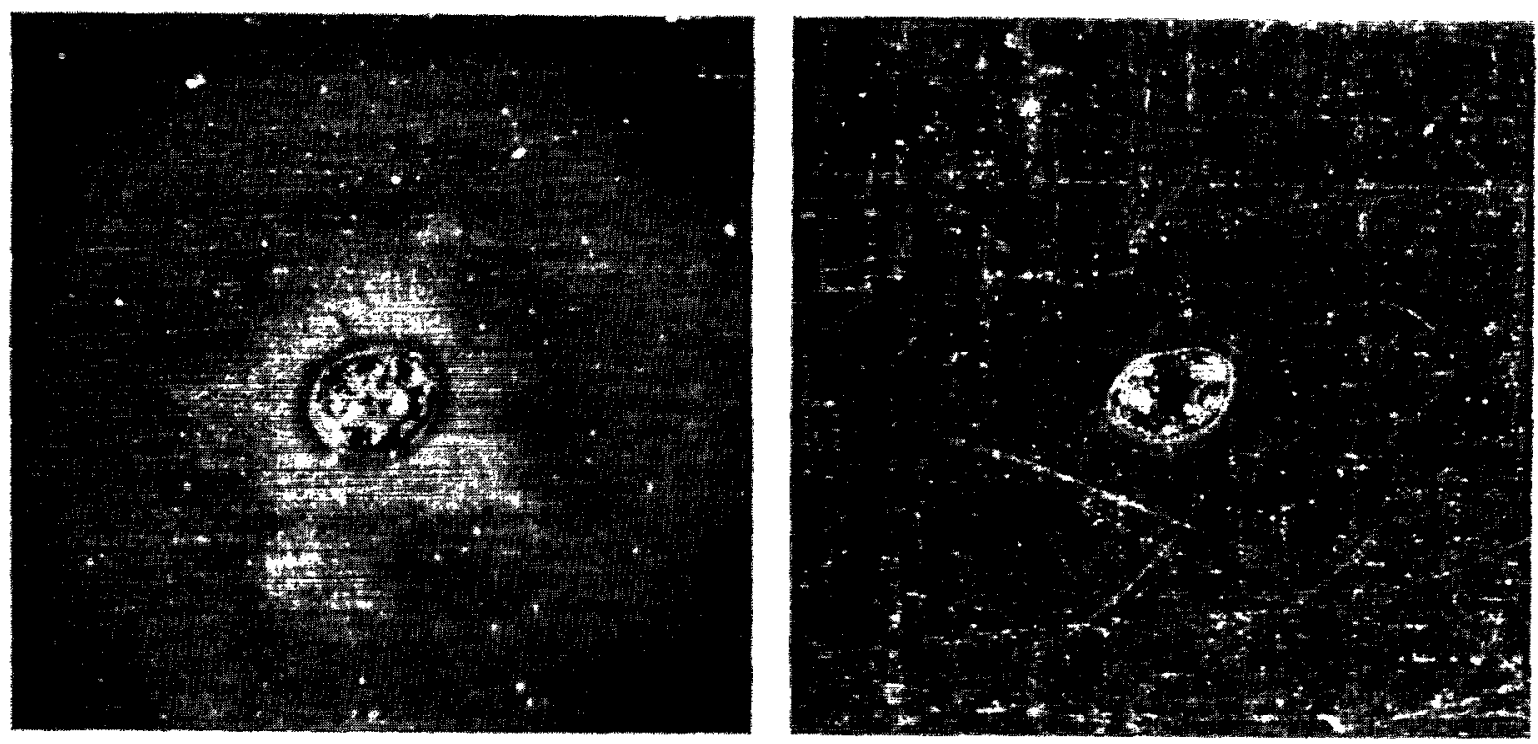

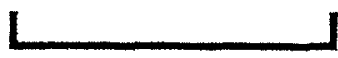

$1 \mathrm{~mm}$

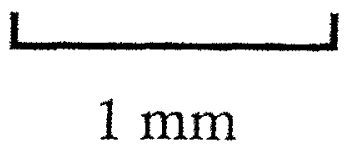

FIG. 6. Drilled holes in (a) 0.025 -mm-thick and (b) 0.127 -mm-thick copper in atmospheric pressure air. 
Machining is difficult with copper due to the high thermal conductivity which results in the rapid loss of heat from the irradiated area. The fact that drilling was only observed in an air environment is due to the metal-oxide layer formed on the surface of the foil. As the foil is heated, the copper reacts with oxygen in the air to form ${ }^{13}$ cupric oxide (CuO) and cuprous oxide $\left(\mathrm{Cu}_{2} \mathrm{O}\right)$. This is observed in Figs. 6(a) and 6(b) where the darkest outer region around the hole is cupric oxide (blue-black) while the next inner discolored band (red) is the cuprous oxide. This colordependent absorption with green-yellow light has previously been seen in the machining of polymers. ${ }^{12}$ When a sufficient metal-oxide layer has formed on the surface, the absorptivity increases and the foil rapidly heats, followed by drill through.

Drilling of aluminum foils in argon was unsuccessful; no machining was evident on the surface of the foil. Limited Al drilling was also observed in air. Holes that were drilled in the aluminum foil required little time $(\sim 10 \mathrm{~s})$ with the hole size being much smaller $(\sim 0.1 \mathrm{~mm})$. At other irradiation spots on the same sample, no machining was observed. This difference is attributed to a nonuniform aluminum-oxide layer on the surface of the foil. Previous work has shown the importance of the metal-oxide layer in the machining of aluminum plates with $\mathrm{CO}_{2}$ lasers. ${ }^{14}$ Without a substantial aluminum-oxide layer, machining is difficult due to the high reflectivity ( $>92 \%$, from Table II) of aluminum to the green-yellow light of the copper vapor laser. $^{7}$

\section{ACKNOWLEDGMENTS}

This research is supported by the National Science Foundation (CTS-9108971). ISL has a National Science Foundation Graduate Fellowship. We acknowledge the DOE for the copper vapor laser. The authors thank $R$. Spears and W. McColl for technical assistance and $\mathbf{R}$. Lindley and $\mathrm{H}$. Ching for helpful discussion.

${ }^{1}$ D. J. Hoffman and L. R. Migliore, Manuf. Eng. 102, 44 (1989).

${ }^{2}$ D. Nelson, Prod. Eng. 33, 16 (1986).

${ }^{3}$ C. D. Rose, Electronics 58, 49 (1985).

${ }^{4}$ R. W. Dreyfus, J. Appl. Phys. 69, 1721 (1991).

${ }^{5}$ B. Braren and A. Afzali, SPIE Proc. 1856-23, 1993.

${ }^{6}$ H. S. Kim, Y. Domankevitz, H. S. Kwok, and J. A. Copley, Appl. Phys. Lett. 55, 726 (1989).

${ }^{7}$ J. H. Weaver, C. Krafka, D. W. Lynch, and E. E. Koch, Optical Properties of Metals (Karlsruhe, Germany, 1981).

${ }^{8}$ A. D. McQuillan and M. K. McQuilllan, Titanium (Academic, New York, 1956).

${ }^{9}$ A. M. Prokhorov, V. I. Konov, I. Ursu, and I. N. Mihailescu, Laser Heating of Metals (Adam Hilger, Rristol, England, 1990).

${ }^{10}$ H. S. Carslaw and J. C. Jaeger, Conduction of Heat in Solids, 2 nd Ed. (Oxford, Cambridge, 1959).

${ }^{11}$ J. F. Ready, Effects of High-Power Laser Radiation (Academic, New York, 1971).

${ }^{12}$ P. L. G. Ventzek, R. M. Gilgenbach, C. H. Ching, R. A. Lindley, and W. B. McColl, J. Appl. Phys. 72, 3080 (1992).

${ }^{13}$ E. G. West, Copper and Its Alloys (Wiley, New York, 1982).

${ }^{14}$ E. Armon, M. Hill, I. J. Spalding, and Y. Zvirin, J. Appl. Phys. 65, 5003 (1989). 